\title{
60 Jahre Deutscher Juristinnenbund
}

\section{Stimmen der Bundesvorstandsmitglieder}

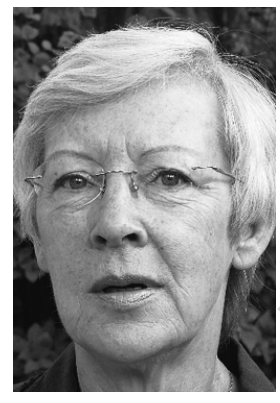

Adelhaid Brandt

Beisitzerin im djb-Bundesvorstand (19931999); Chefredakteurin Neue Justiz, Berlin

Die mit dem Fall der Mauer einhergehenden stürmischen Entwicklungen erforderten für uns Ostdeutsche nicht nur eine Neuorientierung, sie führten auch $\mathrm{zu}$ interessanten Begegnungen mit Personen der eigenen Profession. Auf diese Weise lernte ich frühzeitig so engagierte Juristinnen wie Adelheid Koritz-Dohrmann, Ursula Raue und Karin Schubert kennen und schätzen. Es war daher nur folgerichtig, dass ich in den djb eintrat.

Als erste Juristin aus den neuen Bundesländern wurde ich 1993 in den Bundesvorstand gewählt, dem ich bis 1999 als Beisitzerin angehörte. Aufgrund meiner beruflichen Tätigkeit zeichnete ich für die Presse- und Öffentlichkeitsarbeit verantwortlich.

Diese Arbeit hat außerordentlich viel Spaß, aber auch Mühe bereitet. Denn der Vorstand hatte sich zum Ziel gesetzt, die Präsentation des Verbandes nach innen und außen nachhaltig zu verbessern und die Effizienz der Sacharbeit durch Neukonzipierung der Öffentlichkeitsarbeit zu steigern. Dazu gehörten unter anderem ein Verbandslogo, die Einrichtung einer eigenen Homepage im Internet, einheitliches Briefpapier für die Landes- und Regionalgruppen, Schautafeln, die Umgestaltung der bislang unter der Ägide von Dr. Monika Zümstein als Blätter erscheinenden „aktuellen informationen“ $\mathrm{zu}$ einer Verbandszeitschrift und die Herausgabe einer eigenen Schriftenreihe.

All dies sind heute Selbstverständlichkeiten, sie prägen das seiner Bedeutung nach angemessene Erscheinungsbild des djb; damals aber betraten wir Neuland mit all den dabei auftretenden logistischen und technischen Schwierigkeiten.

Während meiner Zugehörigkeit zum Bundesvorstand konnte ich auch über im Zusammenhang mit der Rechtsangleichung stehende Probleme informieren und auf Benachteiligungen von Frauen in den neuen Ländern aufmerksam machen.

In diesen für mich erfahrungsreichen Jahren habe ich vor allem eines gelernt: Der Kampf um die tatsächliche Gleichstellung von Frauen und Männern bleibt eine stete Pflichtaufgabe.

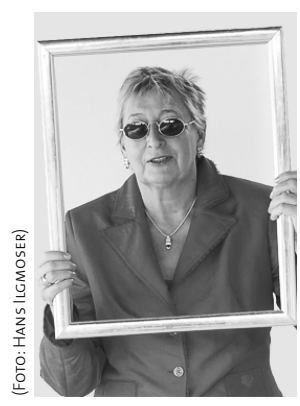

\section{Renate Damm}

Mitglied des erweiterten Bundesvorstands (1979-1981); 2. Vorsitzende des djb (1981-1983); 1. Vorsitzende des djb (19831989), Vorsitzende der Satzungskommission zur generellen Überarbeitung der Satzung (1985); Rechtsanwältin, Hamburg

Als ich 1983 den Vorsitz übernahm, hatte der djb etwas mehr als 500 Mitglieder. Nach meiner sechsjährigen Amtszeit hatte sich die Mitgliederzahl mehr als verdoppelt. Sicherlich hat dazu auch die Tatsache beigetragen, dass ich als Justitiarin des Axel Springer Verlages über einige Pressekontakte verfügte. Ich wünsche mir, dass es selbstverständlich wird, dass jede Juristin Mitglied im djb ist!

In meine Amtszeit fällt auch die Schaffung unserer ersten Geschäftsstelle in Bonn mit Dr. Hertha Engelbrecht als ehrenamtlicher Geschäftsführerin. Wir waren darauf sehr stolz!

Genauso stolz können wir darauf sein, dass der djb schon seit 1983 das Thema der Humangenetik intensiv behandelte und dem Gesetzgeber (warum eigentlich nicht „der Gesetzgeberin“?) damit wichtige Vorleistungen erbrachte.

$\mathrm{Zu}$ meinen persönlichen Highlights gehört mit Sicherheit der gemeinsame Auftritt von Dr. Lore Maria Peschel-Gutzeit und mir vor dem Bundesverfassungsgericht zu der Frage, ob ein Kind einen Anspruch darauf hat, von der Mutter den Namen seines Vaters zu erfahren.

$\mathrm{Zu}$ meinem persönlich wichtigsten Erfolg zähle ich, dass Frauen nunmehr im Falle einer Wiederverheiratung einen früheren Ehenamen beibehalten können und nicht einen Doppelnamen führen müssen. Das war schon ein harter Kampf!

Wenn aller guten Wünsche drei sind, dann wünsche ich mir und allen Kolleginnen, dass Frauen in Führungspositionen zur Normalität werden und die Gleichberechtigung in den Köpfen auch der Männer endlich ankommt. Wir Frauen dürfen allerdings nicht nachlassen, unsere Gleichbehandlung einzufordern. Mein Appell gilt den jungen Kolleginnen: bitte nicht zurücklehnen und davon ausgehen, dass die Männer die Gleichberechtigung auch im Beruf akzeptieren! 


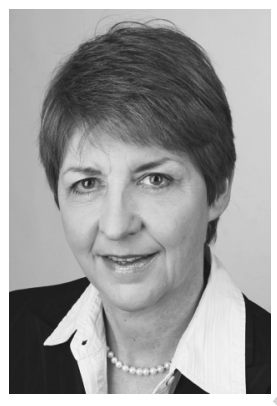

\section{Margret Diwell}

Stellv. Vorsitzende des djb (1999-2001); Präsidentin des djb (2001-2005); Rechtsanwältin, Fachanwältin für Familienrecht in Berlin, Präsidentin des Verfassungsgerichtshofs Berlin

Deutschland hat eine Bundeskanzlerin und sie macht ihre Arbeit gut. Deutschland hat eine Kandidatin für das Amt des Bundespräsidenten und sie ist höchst präsidiabel. Es gibt ein paar herausragende Frauen in Führungspositionen der Wirtschaft, einige Professorinnen an den Universitäten, ein Drittel des Bundeskabinetts ist weiblich, ein Viertel der Verfassungsgerichte hat Präsidentinnen, wobei der Präsident des Bundesverfassungsgerichts gerade öffentlich bedauert hat, dass er keine Richterinnen in das Amt einführen darf, und die Justiz wird von einer sichtbaren Anzahl von Frauen repräsentiert. Eine winzig kleine Bewegung auf einem schier endlos langen Weg zur Verwirklichung der Gleichberechtigung - oder Zufälligkeiten der Statistik? Ausdruck eines gesellschaftlichen Konsenses, der Frauen und Männern die gleiche Teilhabe an Macht, Geld, Erfolg und Familienarbeit gewährleistet, an dem wir nur geduldig weiterpuzzeln müssen, damit in ein oder zwei Generationen überall Geschlechterparität gewahrt ist? Ich meine nein! Es ist der individuellen Durchsetzungsfähigkeit, dem nahezu selbstmörderisch anmutenden Durchsetzungswillen einzelner Frauen (und dem Idealismus der sie fördernden Frauen und Männer) zu verdanken, dass in unserer Gesellschaft auch manchmal Frauen in Führungspositionen sichtbar sind. Dafür zahlen diese Frauen und ihre Familien einen (zu) hohen Preis und eben das unterscheidet die Frauen- von den Männerkarrieren: Männer in Führungspositionen sitzen in einem warmen Kokon von weiblicher Fürsorge, gut gepolstert zudem von einem Steuerrecht, das das Modell Alleinverdienerehe völlig unangefochten trägt, und nun auch noch flankiert von dem brandneuen Unterhaltsrecht, das vorhersehbar und von den Frauen in den Gesetzgebungsgremien deshalb heftig bekämpft ausschließlich zu Lasten der Frauen geht. Eine Gesellschaft, die über 50-jährige Frauen nach 20 bis 30 Jahren Familienarbeit auf den Arbeitsmarkt verweist, ist schlicht ungerecht. Dies hat nichts mit Gleichberechtigung zu tun, aber sehr viel mit dem Frauenbild des Gesetzgebers und angesichts der Fülle der unbestimmten Rechtsbegriffe auch etwas mit dem Frauenbild der Gesetzesanwender(-innen)! Wie leben Familien mit mehreren Kindern zukünftig? Vollzeitbeschäftigte Eltern für ein einträgliches Familieneinkommen und zum Ausschluss von Unterhaltsrisiken. Was ist das für eine inhumane Gesellschaft, die damit jungen wie älteren Frauen das volle Risiko für das Funktionieren der Familien und den Wohlstand dieses Landes aufbürdet? Wo bleibt das Bedürfnis nach Privatheit, gemeinsamer Freizeit, kulturellen Genüssen oder Faulenzen, geschweige denn ehrenamtliches Engagement oder politische Betätigung bei einem zwischen Kindern und Beruf minutiös aufgeteilten Tag? Die Rede ist hier von den Rechten der Frauen. Die in einer solchen Gesellschaft niemals einzulösenden Rechte der Kinder mögen woanders beschrieben werden. Ich verstehe Frauen, die sich auf Knien anflehen lassen, höherwertige Aufgaben zu übernehmen und dankend ablehnen. Ich verstehe, warum trotz besserer Schul- und Hochschulabschlüsse der Frauen seit vielen Jahren Chefsessel das Ziel der allerwenigsten und berufliche Nischen mit erträglichen Arbeitszeiten gesucht sind. Beruf und Karriere sind weniger denn je mit rechtlichen, tatsächlichen und wirtschaftlichen Verhältnissen vereinbar. Ohne ein Mindestmaß an Sicherheit wird sich keine Frau mehr (mehr als) ein Kind wünschen. Übernimmt Mann nicht endlich die Hälfte der Familienarbeit und sorgt der Gesetzgeber nicht für einen fairen Ausgleich für Kindererziehungszeiten (nicht nur im Unterhaltsrecht), verpflichtende und flexible Arbeitsbedingungen ohne Lohn- oder Karriereeinbußen in der Kindererziehungsphase - und die ist eben länger als drei Jahre! - und für Umverteilung der staatlichen Ressourcen in qualifizierte Erziehung und Förderung von Kindern und Jugendlichen, wird es keine Gleichberechtigung und Gleichstellung der Frau in allen gesellschaftlichen Bereichen geben.

Die Vereinsziele des djb sind noch lange nicht erfüllt. Ich wünsche mir für die nächsten 60 Jahre starke Frauen, die dem Fortschritt namens Schnecke Beine machen!

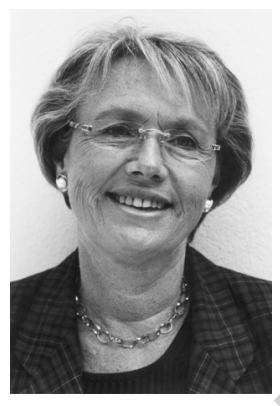

Dr. h.c. Renate Jaeger

Mitglied des erweiterten Bundesvorstands (1983-1987); Beisitzerin im Bundesvorstand des djb (1987-1989); Richterin am Europäischen Gerichtshof für Menschenrechte, Straßburg

Der djb ist der Verband, in dem ich die meisten Gleichgesinnten gefunden und Freundschaften geschlossen habe. Das get together mag ich bis heute nicht missen.

Die Schlagworte der heutigen Terrorismusdebatte erinnern mich an meine Vorstands- und Kommissionstage: Die Voraus- setzung der Freiheit ist Sicherheit. Frauen können ihren Lebensweg zwischen den Anforderungen Familie und Berufswelt nur finden, wenn ihre freie Entscheidung nicht durch unzulängliche soziale Sicherheit bis zur Selbstaufgabe gefährdet ist.

Die eigenständige Sicherheit der Frau wurde nicht erreicht, obwohl der djb mit dem BVerfG damals einen guten Verbündeten hatte. Hervorragend ausgebildete Frauen leben weiterhin prekär und wagen den Schritt in die eigene Familie nicht oder sehr spät. Gescheitert sind wir am Besitzstandsdenken der Männer. Aber auch die Männer haben ihren Besitzstand nicht behalten. Kein Grund zur Freude. Alle haben gegen den Wirtschaftsliberalismus verloren. Die für jedwede Sicherheit 
nötige Solidarität - das Einstehen füreinander - wurde aufgekündigt. Heute langt die Rente für keinen und keine mehr, die eine „normale“ und keine Musterbiografie aufzuweisen haben. Die Arbeitslosigkeit führt in die Armut und Kinder sind ein zusätzliches Armutsrisiko. Auch die Frauenbewegung hat sich entsolidarisiert und damit geschwächt.

Ich weiß nicht, ob ich heute noch den Optimismus und die Kraft für Verbandspolitik hätte. Mein Vertrauen in die Politik hat abgenommen, weil immer mehr Regelungen nur noch Symbolwert haben und Handlungsfähigkeit suggerieren sol- len. Die Probleme sind seit Langem klar; Diagnosen haben wir reichlich. Auch Lösungen wären mit gemeinsamer Anstrengung möglich, wenn statt der Feinstellschrauben die Hebel mit dem nötigen Mut umgelegt würden.

Dem djb und allen, die weiter aktiv Frauenpolitik betreiben, wünsche ich die richtigen Verbündeten unter den Mächtigen, Formulierungskunst, die Herz und Verstand erreicht, und so viel Klarheit in der Gedankenführung, dass andere unsere Ideen als eigene verbreiten.

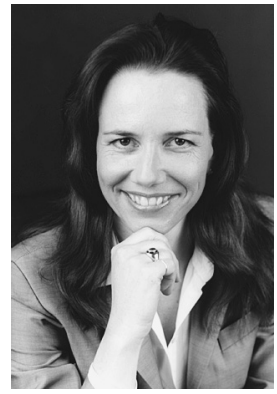

\section{Elisabeth Kotthaus (geb. Müller)}

Beisitzerin im Bundesvorstand (19992001); Vizepräsidentin des djb (20032005); Delegierte des djb bei FIFCJ und FIDA (2001-2005); EWLA-Präsidentin (2001-2005); Hauptverwaltungsrätin bei der EU-Kommission, Brüssel

\section{Der djb als Motor für Juristinnen weltweit}

Eine der in Deutschland weniger stark wahrgenommenen Qualitäten des djb ist seine Vorbildfunktion für Juristinnenvereinigungen weltweit. Meine vier Jahre im Vorstand des djb erlaubten mir einen Einblick in diese Dimension - die sich zwar nicht immer und sofort für den djb unmittelbar auszahlt, wo sich der djb jedoch als Wegbereiter erweist.

Als ein dynamischer Wegbereiter erwies sich der djb auf europäischer Ebene: Der djb organisierte - gemeinsam mit dem britischen Women Lawyers Forum und dem Verein österreichischer Juristinnen - im Jahre 2000 in Berlin den Gründungskongress des Europäischen Juristinnenbunds, der European Women Lawyers Association EWLA (www.ewla.org), deren erste gewählte Präsidentin ich von 2001 bis 2005 war. Nach dem Vorbild des djb beteiligt sich die EWLA regelmäßig an Gesetzgebungsverfahren und mischt sich ein - auf europäischer Ebene. EWLA konnte dadurch sowohl auf den Text des
Verfassungskonvents als auch auf so manche Gleichbehandlungsrichtlinie Einfluss nehmen.

Spannend, faszinierend und horizonterweiternd war auch die Kooperation des djb mit den beiden internationalen Juristinnenvereinigungen FIFCJ (www.fifcj-ifwlc.net) und FIDA (www.fidaweb.org). Beide Organisationen - gegründet 1928 bzw. 1944 - zeigten sich höchst beeindruckt von der Durchschlagskraft des djb. Eine aktive Begleitung von Gesetzgebung, Rechtsprechung und Rechtspraxis auf internationaler Ebene, wie sie der djb betreibt, ist FIDA und FIFCJ zwar bislang noch nicht in einer vergleichbaren Weise gelungen. Dafür aber haben beide Vereinigungen ECOSOC- Status (FIDA seit 1954, FIFCJ seit 1961). So können beide Seiten voneinander profitieren: Der djb von der Möglichkeit, sich jenseits des deutschen Umfelds in die internationale Diskussion einzumischen, und die FIFCJ und FIDA von der Effizienz des djb. Eine der Konsequenzen aus der bisherigen Zusammenarbeit: Die argentinischen Kolleginnen gründeten nach dem Vorbild des djb und der EWLA im Jahr 2005 eine lateinamerikanische Juristinnenorganisation.

Dass der djb 2007 aus FIFCJ sowie FIDA ausgetreten ist, ist daher sehr bedauerlich - nicht nur gehen dem djb Chancen verloren - sondern vor allem enthalten wir Kolleginnen weltweit unsere Unterstützung vor. EWLA und die lateinamerikanische Juristinnenvereinigung belegen, dass der djb eine wichtige Rolle spielen kann. Ich hoffe daher, dass der djb sich bald wieder auf internationaler Ebene engagiert - auch durch Mitgliedschaften bei der FIFCJ und FIDA!

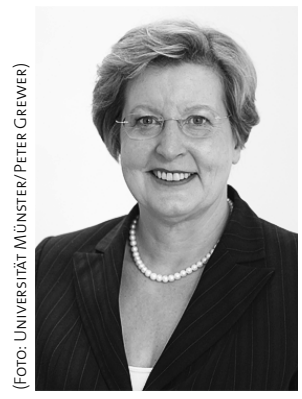

Prof. Dr. Ursula Nelles

Stellv. Vorsitzende des djb (1995-1997); Vorsitzende der Strafrechtskommission (1995-1997); 1. Vorsitzende des djb (19972001); (Kommissarische) Vorsitzende der Kommission Strafrecht (2002-2005); Vorsitzende des Europäischen Juristinnenbundes (EWLA) in Gründung; Hochschullehrerin und Rektorin der Westfälischen Wilhelms-Universität Münster
In besonderer Erinnerung ist mir die 50-Jahr-Feier des djb 1998 in Dortmund geblieben. Das war ein tolles Fest. Absolutes Highlight des Kongresses war die Podiumsdiskussion mit den damals noch lebenden Gründungsmitgliedern des Verbandes. Außerdem war Cherie Booth mit einer Delegation englischer Juristinnen gekommen. Wir haben mit den Britinnen in Dortmund erste Gespräche über die Gründung eines EU-Netzwerks von Juristinnen geführt, das sich im Interesse der Frauen zu Wort meldet. Die Europäische Union war zu einem starken Gesetzgeber geworden, was eine Lobby-Arbeit 
im Sinne der Gleichberechtigung notwendig machte. Die Vorbereitungen zur Gründung des Europäischen Juristinnenbundes (EWLA), die dann 2000 in Berlin stattfand, bestimmten die folgenden zwei Jahre meiner Amtszeit. Unter tatkräftiger Unterstützung der Regionalgruppe Brüssel, die uns mit jeder Menge Informationen, Tipps und Tricks zur Seite stand, habe ich gemeinsam mit Martina Bosch, der damaligen Geschäftsführerin, die Formalien und Anträge zur Verbandsgründung und Förderung bearbeitet und den Gründungskongress vorbereitet.

Die Geschichte des djb ist eine Erfolgsstory. In den vergangenen Jahrzehnten wurden auf Initiative des djb eine Vielzahl rechtlicher Nachteile für Frauen abgebaut. Das waren oft lange und zähe Kämpfe, doch meist hat sich unsere Hartnäckigkeit gelohnt. Ein Beispiel aus meiner Zeit als Vorsitzende der Strafrechtskommission ist die Gesetzesänderung, die auch die Vergewaltigung in der Ehe unter Strafe stellte. Auch im Strafprozessrecht haben wir Erleichterungen für weibliche Opfer erreicht, die vor Gericht als Zeuginnen aussagen müssen. Bei fast jeder Reform haben wir mitgeredet, egal ob es um den Paragrafen 218 oder das Kindschaftsrecht ging.

Dennoch zählen zu den großen Herausforderungen für den djb auch die stagnierenden oder sinkenden Mitgliederzahlen, mit denen sämtliche Verbände, alle ehrenamtlichen Organisa- tionen und NGOs zu kämpfen haben. Es ist heute nicht mehr so, dass man einem Verein beitritt und ewig Mitglied bleibt. Künftig wird daher auch der djb noch deutlicher für seine Mitglieder herausarbeiten müssen, wo der konkrete „Benefit“ einer Mitgliedschaft liegt, ohne jedoch hierbei zu Lasten des Profils und der Außenwirkung des Verbandes die Dienstleistungsfunktion zu stark zu bedienen. Da der Verband so stark ist, weil auch die bedeutenden Juristinnen, ob Ministerinnen oder Verfassungsrichterinnen, Mitglied im djb sind, gilt es auch künftig solche Frauen an den Verband zu binden, damit diese Entwicklung nicht eines Tages abbricht. Manchmal habe ich die Sorge, dass die Idee meiner Vorgängerinnengeneration, die den Verband lange geprägt hat, verloren geht. Für sie war es noch Ehrenpflicht, diesem Verband, der die Zulassung der Frauen zum Jurastudium erkämpft hatte, beizutreten. Man muss sich vor Augen führen, dass die Frauen, die 1949 den Verband gegründet haben, diejenigen waren, die zwischen 1919 und 1932 studiert hatten und die ersten waren, die in den Genuss kamen, das Examen machen zu dürfen. Es wird darauf ankommen, das auf dieser Grundhaltung basierende Verständnis zu Fragen der Gleichstellung und Gleichberechtigung von Frauen bei unseren Mitgliedern wach zu halten und an die nächste Generation von Juristinnen weiter zu geben.

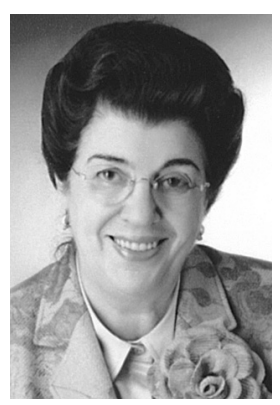

\section{Dr. Lore Maria Peschel-Gutzeit}

Ersatzmitglied des Bundesvorstands (1967-1969): in dieser Zeit setzte sie das „Lex Peschel“ durch, das Beamtinnen und Richterinnen Teilzeiturlaub und Familienurlaub gewährt; Vorsitzende der Kommission Teilzeitarbeit von Beamtinnen (1968); Mitglied des erweiterten Bundesvorstands (1973-1975); Vorsitzende der großen Familienrechtskommission zu den noch offenen Fragen der Familienrechtsreform (1975); 2. Vorsitzende des djb (1975-1977); Vorsitzende der Fachkommission Familienrecht: elterliche Sorge (1976); Vorsitzende der Kommission zur Neuordnung des Rentenrechts (1976); 1. Vorsitzende des djb (1977-1981); Vorsitzende der Familien rechtskommission zur geplanten Änderung des Scheidungs- und Scheidungsfolgenrechts (1983); Vorsitzende der Kommission Jugendhilfe (1984-1989); Vorsitzende der Familienrechtskommission zum Entwurf des Unterhaltsänderungsgesetzes und zum Entwurf eines Gesetzes über weitere Maßnahmen auf dem Gebiet des Versorgungsausgleichs (1985); Vorsitzende der Familienrechtskommission (19881996); Leitung der Unterkommission Unterhaltsrecht (19901992); Vorsitzende der Kommission Anerkennungskultur (2004-2005); seit 2006 Trägerin der Ehrennadel des djb für über 50-jährige Verbandsmitgliedschaft; Vors. Richterin am OLG Hamburg a.D.; Justizsenatorin a.D. in Berlin und Hamburg; Rechtsanwältin in Berlin
Meine Amtszeit als erste Vorsitzende (1977-1981) war von tiefgreifenden sozialen Reformen geprägt: Die sozial-liberale Koalition packte vor allem auf dem Gebiet des Familienrechts heiße Eisen an. Am 1. Juli 1977 trat - nach 20-jähriger Diskussion, an welcher der djb sehr aktiv und mit diversen eigenen Vorschlägen teilgenommen hatte - das erste Eherechtsreformgesetz in Kraft, das das gesamte Familienrecht förmlich auf den Kopf stellte und unter anderem die Familiengerichte einführte. Der djb verteidigte diese Reformen in mehreren Verfahren vor dem Bundesverfassungsgericht aktiv und mit großem Erfolg; ich selbst habe dort als erste Vorsitzende wiederholt und ausführlich plädiert. Ebenso engagiert beteiligte sich der djb durch seine Familienrechtskommission, der ich vorsaß, an der Reform des Rechts der elterlichen Sorge, die schließlich am 1. Januar 1980 in Kraft trat und die das Eltern-Kind-Verhältnis deutlich demokratisierte. Die Familienrechtskommission hatte einen eigenen Reformentwurf formuliert und begründet, welcher im Gieseking Verlag als Buch erschien.

In dieselbe Zeit fiel die Reform des Jugendhilferechts, die schließlich - nach 30-jähriger Diskussion - 1990 verabschiedet wurde und 1991 in Kraft trat. Der djb erarbeitete durch seine Jugendhilfekommission, der ich vorsaß, zwei eigene Reformentwürfe zur Jugendförderung und zur Jugendhilfe, die in Buchform beim Schweizer Verlag veröffentlicht und in die Reformdiskussion des Deutschen Bundestages in vielen Punkten eingegangen sind.

Im Steuer- und Rentenrecht machte der djb durch seine beiden Kommissionen, denen ich angehörte, eigene und sehr neue Regelungsvorschläge, unter anderem zur Einführung der Indi- 
vidualbesteuerung mit Familienadditiven sowie zum Familienlastenausgleich. Zur Absetzbarkeit von Kinderbetreuungskosten bei nur einem Kind verfasste ich für den djb eine gutachterliche Stellungnahme, die mit dazu geführt hat, dass das Bundesverfassungsgericht im Herbst 1977 die Nichtberücksichtigung dieser Kosten im Steuerrecht für verfassungswidrig erklärte. Im Rentenrecht hat sich der djb vor allem und mit großem Erfolg für die eigenständige soziale Sicherung der Frau in der Rentenversicherung eingesetzt; Frau Dr. Kohleiss, meine
Nachfolgerin als erste Vorsitzende ab 1981, und ich sind in diesem Zusammenhang wiederholt vor dem Bundesverfassungsgericht aufgetreten. Mit dem Ende meiner Amtszeit als erste Vorsitzende endete meine Tätigkeit selbstverständlich nicht, die Familienrechtskommission, der ich bis 1991 vorgesessen habe, hat weiterhin diverse eigene Vorschläge erarbeitet, Stellungnahmen vor dem Deutschen Bundestag und vor dem Bundesverfassungsgericht abgegeben, die zum Teil wörtlich in die dann ergangenen Entscheidungen übernommen sind.

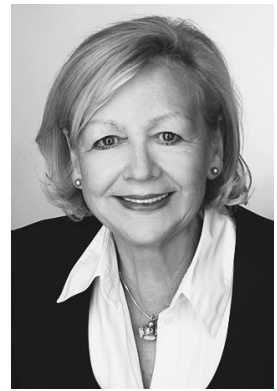

\section{Ursula Raue}

Beisitzerin im djb-Bundesvorstand (19891993); Vorsitzende des Arbeitsstabs DDRKontakte (1990-1993); Vorsitzende des Arbeitsstabs Drogen (1990-1993); 1. Vorsitzende des djb (1993-1997), Vorsitzende des Arbeitsstabs Juristenausbildung (1998-2001); Rechtsanwältin in Berlin

In Weimar wurde ich 1993 erste Vorsitzende des djb, dem Ort der klassischen deutschen Literatur und der Weimarer Nationalversammlung, die im Jahre 1919 dort - im Nationaltheater - die Verfassung der ersten deutschen Republik auf dem Weg brachte. An eben jenem Ort - im Nationaltheater - wurde die Tagung eröffnet. Die Richterin des Bundesverfassungsgerichts Helga Seibert hielt die Festrede, die sich mit der Aussage der Bürgerrechtlerin Bärbel Bohley befasste, die Menschen der DDR hätten in der Bundesrepublik Gerechtigkeit gesucht, aber den Rechtsstaat gefunden.

Weimar 1993 war für jede von uns auf eigene Weise neu und ein Ort behutsamer Annäherungen. Am Ende wurde Schwerin zum Tagungsort für das Jahr 1995 gewählt.

Es gab - wie immer - viel zu tun in diesen Jahren: die Verfassungsdiskussion, das Kindschaftsrecht, die Strafbarkeit einer Vergewaltigung in der Ehe, die Vereinbarkeit von Beruf und Familie, die Quote, das Vergaberecht - um nur einiges zu nennen. Es gab eine neue Kommissionen zum Familienlastenausgleich, eine für die Probleme der älteren Menschen und eine für das Europarecht.

Im Inneren des Verbandes wurden die Regionalgruppen zu Landesgruppen verbunden, was nicht überall Begeisterung auslöste, und der djb war in den neuen Bundesländern präsent.

Als 1994 Professorin Dr. Jutta Limbach zunächst Richterin des Bundesverfassungsgerichts und wenig später dessen Präsidentin wurde, konnten die Expertinnen des djb häufiger juristische Stellungnahmen zu verfassungsrechtlichen Fragen abgeben - eine neue Herausforderung.

Frauen des djb waren auch in vielen der einschlägigen Landesministerien präsent, was für die Durchsetzung frauen- und gleichstellungsrelevanter Forderungen in einzelnen Fällen sehr hilfreich war.

1995 reiste eine Gruppe des djb zur Weltfrauen-Konferenz in Peking, wo sich zu unserem Erstaunen die offizielle deutsche Delegation ein ums andere Mal die Arbeitsergebnisse des djb zu eigen machte, was in der Folge den professionellen Gedankenaustausch beförderte.

Die Mitgliederzahl stieg in den Jahren 1993 bis 1997 kontinuierlich. In Augsburg konnte ich 1997 das 2.500. Mitglied begrüßen.

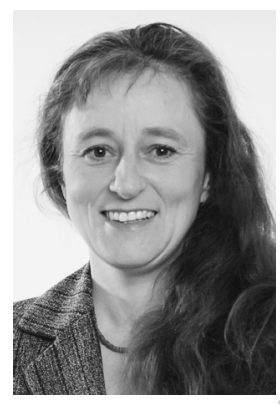

Prof. Dr. Susanne Scheller Beisitzerin im Bundesvorstand (19911993), Vorsitzende des Arbeitsstabs Rechtsfragen der Kinderbetreuung (19931997); Professorin an der Fachhochschule für Polizei in Villingen-Schwenningen

1983 anlässlich der Arbeitstagung des djb in Hamburg bin ich - damals im 2. Semester - eingetreten. Ich war damals wahrscheinlich das jüngste Mitglied. Durch meine Mutter, die als Richterin i.R. zur gleichen Zeit mit mir eintrat, wurde ich auf den djb aufmerksam.

Von Anfang an hat der djb für mich eine Art „MentoringFunktion“ erfüllt. Ich hatte dort Vorbilder wie zum Beispiel
Jutta Limbach, Lore-Maria Peschel-Gutzeit, Antje Sedemund, Renate Damm, Annelies Kohleiss, Adelheid Koritz-Dohrmann ... Bei den Arbeitstagungen, die ich regelmäßig besuchte, lernte ich Frauen kennen, die es als Juristin bis ganz nach oben geschafft haben. Dies war mir ein großer Ansporn, gleichzeitig hat es mich aber auch etwas unter Druck gesetzt, in meinem Studium ebenfalls die Erwartungen zu erfüllen, die an eine junge Kollegin gestellt waren.

Im Herbst 1985 begann ich eine Untergruppe an meinem Studienort in Freiburg aufzubauen und organisierte bis etwa 1990 gemeinsam mit der Richterin am OLG Herta Mattes monatliche Veranstaltungen. 1991 bei der Arbeitstagung in Lübeck wurde ich als jüngstes Mitglied in den Bundesvorstand gewählt, mit der besonderen Aufgabe, mich um die Belange junger Mitglieder zu kümmern. Ich startete eine Umfrage zu 
den Bedürfnissen und Wünschen der Mitglieder unter 40. Dies führte unter anderem zur Gründung des Arbeitsstabes „Berufsorientierung und Karriereplanung“. 1993-1997 leitete ich den Arbeitsstab „Rechtsfragen der Kinderbetreuung“, der eine Stellungnahme des djb zur Umsetzung des Rechtsanspruchs auf einen Kindergartenplatz und eine Musterklage erarbeitete.

Einen nichtjuristischen Beitrag zum Leben des Verbandes konnte ich bei der Arbeitstagung 1987 in Hannover einbringen. Ich durfte die Kolleginnen beim abendlichen Beisammensein mit orientalischem Tanz unterhalten. Heike Mundzeck hat dies in ihrem Film "Juristinnen in Deutschland“ aufgegriffen.

Der djb hat mein Selbstverständnis als Juristin und berufstätige Frau nachhaltig geprägt. Nach einer 6-jährigen Phase als Rechtsanwältin und Fachanwältin für Familienrecht bin ich seit 2002 Professorin an der Fachhochschule für Polizei in Villingen-Schwenningen (Baden-Württemberg). Die flexibleren Arbeitszeiten kommen meiner Familie zu Gute. Ich habe zwei Töchter im Alter von 6 und 1 1/2 Jahren.

Seit wenigen Monaten habe ich in der Hochschule das Amt der Gleichstellungsbeauftragten inne und kann nun auf vieles aus meiner Arbeit im djb zurückgreifen. Derzeit entwickle ich für die Hochschule ein Konzept „Vereinbarkeit von Familie und Studium“.

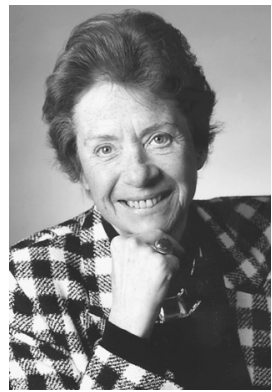

\section{Antje Sedemund-Treiber}

Mitglied des erweiterten Bundesvorstands (1985-1987); 2. Vorsitzende des djb (1987-1989); 1. Vorsitzende des djb (19891993), Vorsitzende des Arbeitsstabs Beamtenversorgung (1990-1993); Vorsitzende des Arbeitsstabs Deutsch-deutsche Rechtsbeziehungen (1990-1993); Vorsitzende der Verfassungskommission (19921993); Präsidentin des BPatG i.R., Bonn

Von 1989 bis 1993 habe ich den djb geleitet. Dies waren mit der Wiedervereinigung geschichtsträchtige und aufregende Jahre, die am djb nicht vorbeigegangen sind. Außer der traditionellen Arbeit des Verbandes insbesondere auf den Gebieten des Gleichstellungs-, Familien- und Strafrechts waren vorrangig zwei durch die Wiedervereinigung bedingte Komplexe zu bewältigen.

Der erste betraf den Abschluss der Arbeiten an der unendlichen Geschichte einer Reform des „\$218“. Während in der DDR mit einer Fristenlösung ohne Beratungspflicht eine liberale Regelung galt, war der westdeutsche Gesetzgeber mit einem in allen Teilschritten umstrittenen Indikationenmodell mit Beratungspflicht befasst, das wiederholt auch das Bundesverfassungsgericht beschäftigte. Mit der Wiedervereinigung musste eine gesamtdeutsche Lösung gefunden werden. Sie wurde bei den Verhandlungen über den Einigungsvertrag hochstrittig diskutiert. Schließlich einigte man sich auf eine befristete Fortgeltung der beiden unterschiedlichen Systeme im Bereich der früheren DDR einerseits und Westdeutschlands andererseits. Die Erarbeitung einer einheitlichen gesamtdeutschen Lösung wurde dem Gesetzgeber bis Ende 1992 aufgegeben. Sie führte zu dem heute maßgebenden Recht. In die Arbeiten für diese Neuregelungen, die einen parteiübergreifenden runden Tisch von Vertreterinnen aller Parteien umfasste, war der djb intensiv eingeschaltet. Er hat einen eigenen Gesetzentwurf erarbeitet und vorgelegt, dessen Überlegungen zum Grundkonflikt des Schwangerschaftsabbruchs zu dem Besten gehört, das zu diesem Problem geschrieben worden ist. Weitere Einzelheiten an dieser Stelle darzulegen würde zu weit führen. Sie sind in unserer Broschüre "Juristinnen in Deutschland, Die Zeit von 1900 bis 1998 " nachzulesen.

Der zweite durch die Wiedervereinigung bedingte Schwerpunkt unserer damaligen Arbeiten betraf das Verfassungsrecht. Änderungen des Grundgesetzes, die sich aus der Wiedervereinigung ergaben, sollten nach einer Entscheidung des Einigungsvertrages innerhalb von zwei Jahren abgeklärt werden. Eine breite verfassungsrechtliche Diskussion war die Folge. Änderungsvorschläge für unseren Verband hat der djb in einer ad hoc eingesetzten Verfassungskommission erarbeitet und vorgelegt.

Schließlich zeichnete sich in diesen Jahren ein weiteres Problem ab, das nicht durch die Wiedervereinigung bedingt war, sondern allgemeine gesellschaftspolitische Ursachen hat: der wachsende Anteil älterer und alter Menschen an der Bevölkerung. Dieser Anteil nimmt bekanntlich ständig zu. Die damit verbundenen Probleme sind vielfältig, sehr sensibel und zum Teil noch wenig erkannt, jedenfalls nicht hinreichend reflektiert. Frauen machen nicht nur einen großen Teil dieses Bevölkerungskreises aus. Sie haben auch ein besonderes Gespür für hier bestehende Probleme und scheuen nicht davor zurück, diese anzusprechen und sich mit ihnen auseinanderzusetzen. Es war für mich deshalb ein nobile officium, dass der djb auch in diesem - nicht von den traditionellen Problemen der Gleichstellung gekennzeichneten - Bereich sein Wissen und Können einbringt, einfach gesagt: sich engagiert. Wir haben deshalb die Kommission Ältere Menschen gegründet, die inzwischen zu vielen Einzelfragen Stellung genommen hat, nicht zuletzt zu der sensiblen, in der Öffentlichkeit bisher kaum beachteten Frage wie der „Gewalt gegen alte Menschen“. In der Arbeit der Kommission „Ältere Menschen“ sehe ich eine wichtige Zukunftsaufgabe unseres Verbandes.

Jenseits aller Sacharbeit haben wir uns mit dem „Innenleben “ des djb befasst. Seit Mitte der 80er Jahre war der Verband stark gewachsen. Das erforderte einerseits neue transparente Strukturen für die Arbeitseinheiten des Verbandes. Jedes Mitglied sollte wissen, wie diese aufgebaut sind und auf welche Weise es sich dort einschalten kann. Um geeignete, sicher verbesserungsbedürftige Lösungen haben wir uns bemüht. Andererseits wollten wir Wege finden, die Mitglieder besser 
als durch die bis dahin üblichen Rundschreiben über Tagungen des djb, über die Arbeit, aber auch über das Leben des Verbandes zu unterrichten. Nicht zuletzt haben wir an solche Mitglieder gedacht, die eine Untergruppe - heute Regionalgruppe - nicht erreichen, um Informationen über den djb zu erhalten. „Monis Blättchen“ wurde geboren. Wissen Sie noch, was das ist? Kaum. Dr. Monika Lanz-Zumstein war damals für Öffentlichkeitsarbeit unseres Verbandes zuständig. Sie hatte es übernommen, eine regelmäßige Information - die ge-

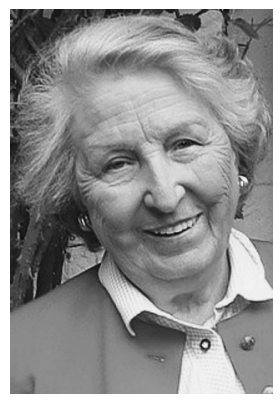

\section{Dr. Helga Stödter}

Schriftführerin im djb-Bundesvorstand (1967-1969), Mitglied des erweiterten djb-Bundesvorstands (1969-1975) seit 2005 Trägerin der Ehrennadel des djb für über 50-jährige Verbandsmitgliedschaft; Rechtsanwältin, Hamburg

Als ich Anfang der 50er Jahre - als Anwaltsassessorin - dem Juristinnenbund beitrat, ahnte ich nicht, welche Rolle er in meinem späteren Leben und bis zum heutigen Tage spielen würde. Erster Höhepunkt war 1950 meine Teilnahme am Juristentag in Frankfurt am Main, als Erna Scheffler ihre Thesen für ein neues Familienrecht erfolgreich vortrug. Nur beim Namensrecht protestierten die Kollegen!

Die folgenreichste Mitarbeit in einer Kommission war für mich jene zur „Reform des Unehelichenrechts“ unter dem Vorsitz von Maria Kramer-Schulz. Wir waren nur zu dritt. Anneliese Cuny war dabei. Ich war Berichterstatterin. Die meisten unserer Forderungen wurden später Gesetz.

Acht Jahre Vorstandsarbeit im djb waren kämpferische Jahre! Das ganze Familienrecht musste dem Grundgesetz angepasst werden - zum Teil gegen erbitterten Widerstand. Der djb fühlte sich vor allen anderen verantwortlich, für die Rechte der Frauen und Mütter einzutreten.

Der djb war Mitglied in der FIDA, dem internationalen Dachverband nationaler Juristinnenverbände. Das erste Mal nach dem Kriege sollte eine Deutsche Präsidentin werden. Weil nauen Zeitabstände erinnere ich nicht mehr - für alle Mitglieder unseres Verbandes zu erstellen. Mit viel Arbeit, wenig Geld und reichlich Charme hat sie dies auf den Weg gebracht. Zwei doppelt bedruckte Seiten, geheftet mit einer Klammer, waren es nach meiner Erinnerung am Anfang. Heute ist daraus unsere ebenso ansehnliche wie informative Verbandszeitschrift geworden.

Insgesamt waren es ausgefüllte und menschlich wie fachlich sehr bereichernde Jahre.

die Wunschkandidatinnen - Renate Lenz-Fuchs und Maria Kramer-Schulz - aus privaten und beruflichen Gründen ablehnten, wurde ich vom djb vorgeschlagen und 1973 in Penang/Malaysia zur Präsidentin gewählt. 1975 war dann der FIDA-Kongress in Hamburg, bei dem es um die „Rechte der Frauen in Familie, Beruf und Gesellschaft" ging. Viele Kolleginnen halfen bei den Vorbereitungen - mit Erfolg.

1972 wurde ich in die Ständige Deputation des Deutschen Juristentages gewählt. Unter anderem übernahm ich 1974 beim Juristentag in Hamburg den Vorsitz der Arbeitsrechtlichen Abteilung. Die meisten unserer Beschlussempfehlungen zur „Chancengleichheit der Frauen im Beruf“ wurden später zu Gesetzen. Wiederum aktuell: 1982 diskutierten Hertha Engelbrecht und ich bei der Schlussveranstaltung über ein Antidiskriminierungsgesetz (!), übrigens kontrovers. In den Jahren danach wurde die Förderung von Frauen für Führungspositionen (so auch der Name der Stiftung, die ich 1988 errichtete) zum Mittelpunkt meiner ehrenamtlichen Tätigkeit und ist es noch. Ich bin davon überzeugt, dass wir in Wirtschaft und Gesellschaft schon jetzt und noch mehr in Zukunft qualifizierte Frauen brauchen.

Wenn ich zurückblicke, dann sehe ich uns als Pionierinnen. Wir waren erfolgreich, weil wir gemeinsame Überzeugungen hatten und zusammenhielten. Aus Kolleginnen wurden Freundinnen. Es waren sinnvolle Jahre. Das wünsche ich auch der jüngeren Generation: gemeinsame Überzeugungen und die Entschlossenheit, sie solidarisch durchzusetzen und, auch dadurch, den Sinn unserer Aufgaben als Juristinnen zu erleben.

\title{
Neue Pressemitteilungen und Stellungnahmen
}

\author{
http://www.djb.de/stellungnahmen-und-pressemitteilungen/
}

\author{
Pressemitteilungen \\ o8-10 3. Bilanz Chancengleichheit - Stagnation auf nied- \\ rigem Niveau. Juni 2008. \\ 08-09 Netzwerk CEDAW-Alternativberichterstattung prä- \\ sentiert Forderungen in New York. Juni 2008. \\ 08-08 Netzwerk der Antidiskriminierungsorganisationen in \\ Deutschland wendet sich an EU-Kommissionspräsi- \\ dent José Manuel Barroso. Juni 2008.
}

\section{Stellungnahmen}

08-12 Zum Entwurf eines Gesetzes über genetische Untersuchungen bei Menschen (Gendiagnostikgesetz GenDG) vom 30. Juni 2008. 